\title{
A Study on the Architectural Plan for Revitalization of Gap in Boundary Space - focusing on Ilshin Textile Factory and Jeonnam Textile Factory Site Utilization Plan
}

\author{
Sue Jung Go ${ }^{1}$, Donghyeog Choi ${ }^{2}$ \\ ${ }^{1}$ Undergraduate Student, Dept. of Architecture, Gachon University, Korea, rhtnwjd1312@naver.com \\ ${ }^{2}$ Associate Professor, Dept. of Architecture, Gachon University, Korea, donghyeog@gachon.ac.kr
}

Corresponding author: Donghyeog Choi

\begin{abstract}
This study is aimed to propose an architectural plan to revitalize the disconnected space in the process of urban development focused on the spatial and temporal gap between the past and development. To achieve this, it intends to allow the gap space between spaces to play a role as a space with new value for the disconnected space that occurred during the process of urban development. The contents of this study are as follows. First, the concept and characteristics of space and boundaries and gaps between spaces are identified. Second, the typological characteristics of the gap space are known. Third, an architectural plan that can be applied according to the typological characteristics of the space of the gap at the proposed site was presented. The proposed architectural plans in this study are summarized as follows. First, this study proposed to divide the space by emptying the existing space and expanding it with a frame for the gap between the large space and the small space. Second, it proposed to expand the space of the gap between the overlapping roofs by using a wire frame. Third, the space between the buildings in the site is proposed as a place to expand the spatial meaning of the gap. The proposal of this study is a planning method that changes the physical properties of the gap space so that the gap space as a boundary space can play a central role in space. In addition, it is a method of planning that expands the meaning of the space of the gap, and can revitalize the proposed site by weaving and combining the spaces that were disconnected from each other. The proposal of this study is to spatially and functionally expand the possibilities of the gap space as a boundary space. The significance of this study lies in the idea that it does not simply extend the physical scale of the space, but suggests a planning method that can fill the temporal and spatial gaps of partially cut off urban spaces.
\end{abstract}

Keywords: Boundary Space, Gap, Expansion, Connection, Preservation

\section{Introduction}

\subsection{The Background and Purpose of the Study}

Space is basically formed by the interaction that occurs between an object and the human who perceives it, and human attitude toward space is a psychological reflection of their visual world[1]. In other words, the subjective world of space can be changed at any time depending on an individual's emotions and psychological state, and the meaning of space is made by reflecting an individual's psychological attitude and sensibility. A boundary is formed between spaces with different meanings, and a space gap exists where the properties of the boundary are revealed. The gap in space can be

Received: August 08, 2021; $1^{\text {st }}$ Review Result: September 25, 2021; $2^{\text {nd }}$ Review Result: November 15, 2021 Accepted: December 31, 2021 
interpreted in various meanings other than spatial, temporal, and abstract concepts, and differences in ideas and perceptions occur due to individual psychological and mental influences. Therefore, the gap space is formed as a frame of perception as an intrinsic image space by psychological interaction through the relationship between the individual and others[2]. A gap exists at the boundary as a small space in the boundary space, and as a passage through all boundaries, it is a space of freedom to move, and it plays the role of mediation rather than a separation between the outside and the inside[3]. In this way, the gap acts as an intermediate zone that forms a new area, and the new value created in the gap can serve as a place to revitalize the existing space.

Recently, cities have grown rapidly, and in the process of urban development, spaces that are being cut off have gradually been created, and a gap has occurred between the spaces of the past and the developed spaces. This study intends to pay attention to the temporal and spatial gaps in urban space that occur along with the development of cities. In particular, this study intends to propose an alternative architectural plan that can revitalize the space of the gap that exists in the boundaries that occurred in the process of city development to play the role of a space with new value.

\subsection{Content of the Study}

This study intends to propose an architectural plan that can revitalize the space between gaps into a place with new value according to its typological characteristics. The contents of this study are as follows. First, it identifies the concept and characteristics of space and boundaries and gaps between spaces. Second, it identifies the typological characteristics of the gap space. Third, in order to create a specific architectural alternative plan that can be activated as a space with new values by spatially expanding the value of the space of the gap, this study proposes an architectural plan that can be applied according to the typological characteristics of the space of the gap at the proposed site.

\section{Theoretical Background}

\subsection{The Meaning of Boundaries as Space}

In general, boundaries refer to ambiguous areas such as two opposing worlds coexisting, cosmos and chaos, everyday and non-routine, center and peripheral, sacred and profane, movement and static, and public and private. In other words, the boundary means the physical and psychological domain shared by two opposing worlds[3]. The modern absolute space has no boundaries, is infinite, and is uniform. However, existential space signified by humans is relative, has boundaries, and has differences. The space of an object is not limited only by the outline of the object itself, but has a spatiality that is meaningful in our lives around, in the vicinity of, and in that direction[4]. In this boundary space, transformation begins and new identities are created as exchanges and communication between unlikely events occur[5]. Therefore, even a similar spatial organization may have different characteristics depending on the specific treatment of the boundaries that define the space. In this way, a boundary is the point where two different areas meet, that is, the point where one ends and the other begins. These boundaries act as boundaries of domains in architecture[6].

The space constitutes a domain through boundaries and creates a place of experience. And the boundary that appears through unit composition has an empirical character. The nature of this boundary is not limited to the inner space, but it draws in the activities of the outside to the inside, blurring the boundary between the inside and the outside[3]. [Fig. 1] is the type of boundary that creates a place of experience. Basically, these four types of boundaries and the boundaries developed through them make up the area where people are active[3]. 

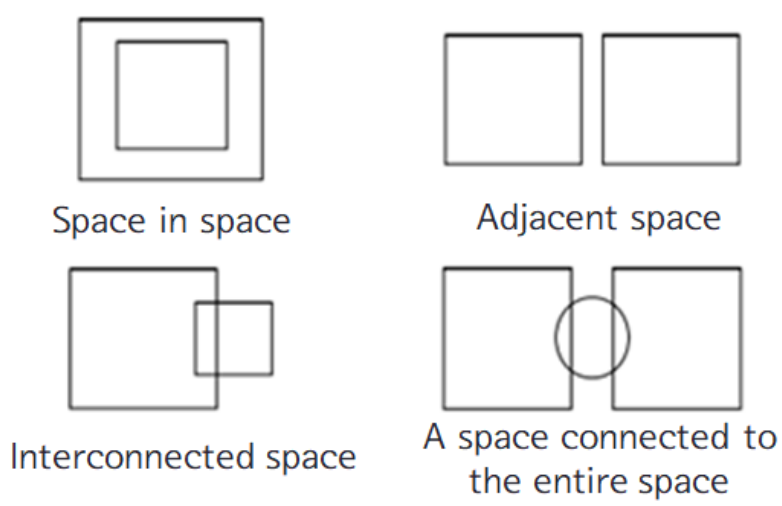

[Fig. 1] Type of Boundary[3]

\subsection{The Gap Space as a Boundary Space}

A gap exists at the boundary as a small space. It can exist wherever space changes its personality or at the boundary where different spaces meet. The gap implies two-sided spatiality as a passage that becomes a boundary between different spaces[7]. If the worlds of areas A and B are separated, there is a spatial gap between them. This gap is a boundary region in the spatial meaning. In this way, the gap as a boundary space can be found in the state where the two worlds are separated from each other at a distance that is not directly affected and are at the same time related to each other. In this case, the gap is a space that encompasses all boundaries and is a transition space that does not cut the space but plays a role of mediation. In architecture, the transition space refers to a connecting space that mediates the exterior and interior, or between space and space[8]. In the architectural space of modern society, the perception of boundaries between interior and exterior, space and form elements is blurring as the previous perception of an independent entity disappears. As a result, modern architects are making various attempts on the physical properties of the surface as a boundary for harm, breaking away from the previously conventional relationship between inside and outside[9]. Architect Sou Fujimoto pursues architecture in between with various possibilities before differentiation. In his architecture, the space between $\mathrm{A}$ and $\mathrm{B}$ is not simply a physical thing that exists in between, but the quality of A and B itself has already changed, and each of them melts into each other to signify a place of smooth change[10]. As such, the space of the gap as a boundary space has the potential to play a role of a spatial center, going beyond a simple mediation space. This study aims to identify the spatial typological characteristics of the void by paying attention to the potential of the void space as a spatial center, and to propose a spatial plan according to the typological characteristic.

\subsection{Spatial Type of Gap Space}

The gap forms an area of a small space and acts as a key element in the area construction process, separating and mediating one area from another. Due to the gap, different spaces are no longer a simple and fixed relationship, but a complex and fluid relationship can be maintained. The relationship between spaces constantly affects each other, and appears as an indeterminate phenomenon that gradually transforms and mutates[11]. The gap between spaces goes beyond the frame of the outline and overlaps through the boundary, and the difference between the overlaps forms a rich space. As the two worlds fall in the form of a boundary area, a spatial gap is created between them, and the space in the gap maintains the nature of the boundary and has an ambiguous area. The spatial types of these gaps are shown in [Table 1][3]. 
[Table 1] Spatial Type of Gap[3]

\begin{tabular}{c|c|c}
\hline $\begin{array}{c}\text { Gaps created by overlapping } \\
\text { spaces }\end{array}$ & Gap due to interlocking overlap & $\begin{array}{c}\text { Gaps in adjacent spaces at a } \\
\text { distance from each other }\end{array}$ \\
\hline $\begin{array}{c}\text { Perceived as a gap between the } \\
\text { form and the boundary space as a } \\
\text { background by the clear difference } \\
\text { in size between the two spaces }\end{array}$ & $\begin{array}{c}\text { Each space secures an area due to } \\
\text { the gap and can control each } \\
\text { other's functional and symbolic } \\
\text { characteristics }\end{array}$ & $\begin{array}{c}\text { Each united space forms an } \\
\text { independent and central place due } \\
\text { to the gap. }\end{array}$ \\
\hline & &
\end{tabular}

The typological characteristics of the space of the gap are summarized as follows. First, the space of the gap due to the overlap of spaces is a space where the external and internal masses are overlapped. That is, a space recognized as a gap occurs due to the clear size difference between the two spaces. Second, the space of the gap due to the interlocking overlap is the space of the gap that occurs in the space between the walls, floors, and ceilings constituting the space overlapping each other. The space of these gaps is not formal and has the characteristics of a mixture of various elements. Third, gaps in adjacent spaces that are distant from each other are spaces formed between buildings and have typological characteristics as spaces between each independent space.

\section{Architectural Plan According to the Typological Characteristics of Gaps}

This study intends to propose an architectural alternative that can be activated as a space with new values by spatially expanding the value of the space of the gap as a boundary area. This study proposes an applicable architectural plan according to the typological characteristics of gaps.

\subsection{Overview of the Proposed Site}

Ilshin Textile Factory and Jeonnam Textile Factory, located in 60 Seorim-ro, Im-dong, Buk-gu, Gwangju, are factory sites that have stopped operating since 2017. The 330,000 $\mathrm{m}^{2}$ site houses huge industrial facilities ranging from dormitories, private houses, childcare and educational facilities, and medical facilities. With urban development, this place was cut off from the city like an island, making it a space of the past with only traces.

[Table 2] Overview of the Proposed Site[12]

\begin{tabular}{l|l|c}
\hline Classification & \multicolumn{1}{c|}{ Contents } & \multicolumn{1}{c}{ Location } \\
\hline Location & 60 Seorim-ro, Im-dong, Buk-gu, Gwangju & $\begin{array}{l}\text { About } 330,000 \mathrm{~m}^{2},(100,000 \text { pyeong) } \\
\text { Ilshin Textile: } 140,000 \mathrm{~m}^{2}, \text { Jeonnam Textile: } 160,000 \mathrm{~m}^{2}\end{array}$ \\
\hline Land Area & $\begin{array}{l}\text { Normal Wood Structure, Reinforced Concrete Structure, } \\
\text { Brick Structured }\end{array}$ & \\
\hline Estructure & 1935 & rablished
\end{tabular}

The proposed site is a space where 4,000 workers worked in the past, so there are densely packed factory buildings. In addition, due to the nature of factory buildings, there are gaps with various 
characteristics between continuous buildings with long masses. The value of space can be expanded through the morphological and functional expansion of the space in these gaps. This study aims to find a method of planning that can revitalize the existing empty space through the space of the expanded gap.

\subsection{The Space of the Gap in the Proposed Site}

Jeonnam Textile Factory and Ilshin Textile Factory are large-scaled textile factories with long factory masses. There are various empty spaces due to these types of factories. In this study, these spaces were defined as spaces of gaps. First, the type of gap created by the overlap of spaces found in the site is a space created by overlapping a large factory mass and a smaller factory mass. This space is a space created by the outer and inner walls, such as corridors. The second type can be found on the roof of the factory remaining on the site. The roofs overlap and interlock to form a gap between the overlapping roofs. Third, there is a gap between the building and the adjacent space formed in the space between the building.

[Table 3] The Space of the Gap in the Proposed Site

\begin{tabular}{c|c|c|c|}
\hline $\begin{array}{c}\text { Gaps created by overlapping } \\
\text { spaces }\end{array}$ & Gap due to interlocking overlap & $\begin{array}{c}\text { Gaps in adjacent spaces at a } \\
\text { distance from each other }\end{array}$ \\
\hline A gap between the space & $\begin{array}{c}\text { A gap between the overlapping } \\
\text { roofs. }\end{array}$ & The space between buildings. \\
\hline $\begin{array}{c}\text { Space in the corridor gap next to } \\
\text { both sides of the large factory mass } \\
\text { and the smaller factory mass } \\
\text { overlap. }\end{array}$ & $\begin{array}{c}\text { The shape of the gap in the factory } \\
\text { caused by the overlapping roof, the } \\
\text { space between the overlapping } \\
\text { roofs. }\end{array}$ \\
\hline
\end{tabular}

\subsection{Architectural Plan Corresponding to the Spatial Type of Gaps}

\subsubsection{Expansion of the Gap between Space}

The space between the large space and the small space can be divided by emptying the existing 
space and expanding it with a frame. The space of this extended gap is a passage through all boundaries and a space of freedom to move, and can act as a space of mediation rather than a separation between the outside and the inside.

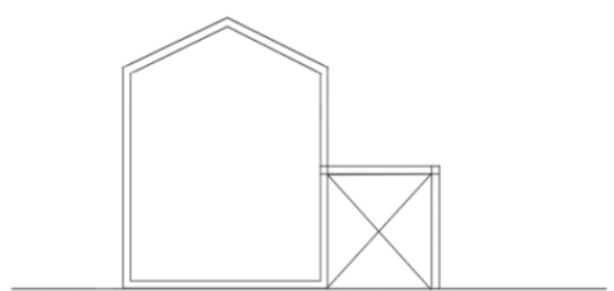

Existing gap space

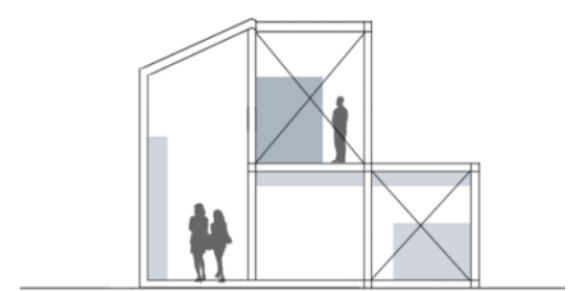

Expanded gap space

[Fig. 2] Expansion of Space According to the Division of the Gap

This study proposes to expand the space of the gap in the production line, which is not currently being used, to the existing space with a frame. The empty space is expanded vertically, and the disconnected wall of the factory serves as an open space using frames. Various cultural programs are planned in the space of this extended gap, and it is expanded into a space of spatial and functional communication.

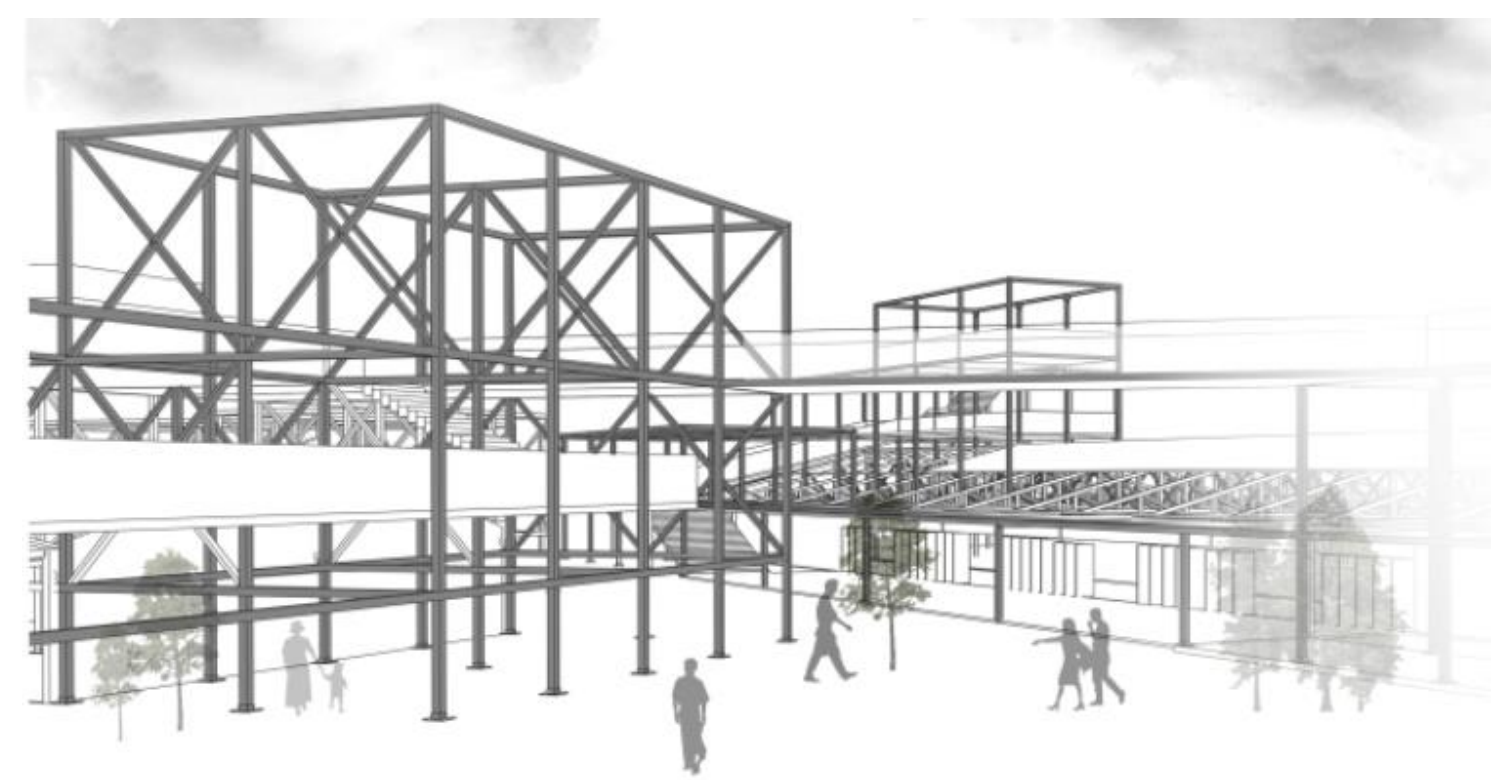

[Fig. 3] Expansion of the Gap as an Open Space - Cultural Creative Street

The cultural creative street in [Fig. 3] was a disconnected space as it was the furthest away from the entrances of the existing Ilshin Textile Factory and Jeonnam Textile Factory. The factory building, which was located the farthest away, is planned as the center of culture, and it is planned to be an open space where many people can visit. The empty space expands vertically, and the frame of the gap digs into the inside of the factory, which was the existing space on the first floor, and expands to the space on the third floor to accommodate various cultural programs.

\subsubsection{Expansion of Gaps by Overlapping}

As a second type of gap, this study proposes to expand the gap space caused by overlapping roofs 
using wire frames. The space of the gap extended by using a wire frame is connected with the existing structure to expand the meaning of the gap as an empty space. This new relationship between the inner and outer space has a positive effect on the environment of the inner space where no light is received, and is an architectural planning proposal that emphasizes the spatial meaning of the existing gap.

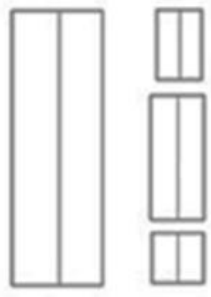

Space between the past

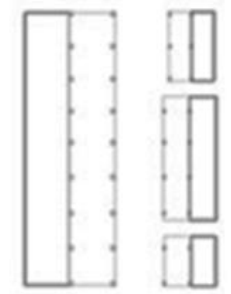

Expanding the space between gaps

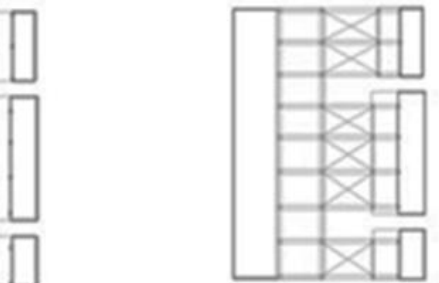

Expansion of empty space

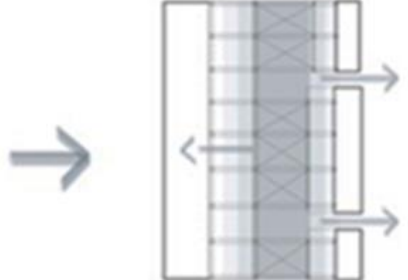

Emphasizing the space between gaps

[Fig. 4] The Space Concept of the Gap Extended by the Wire Frame

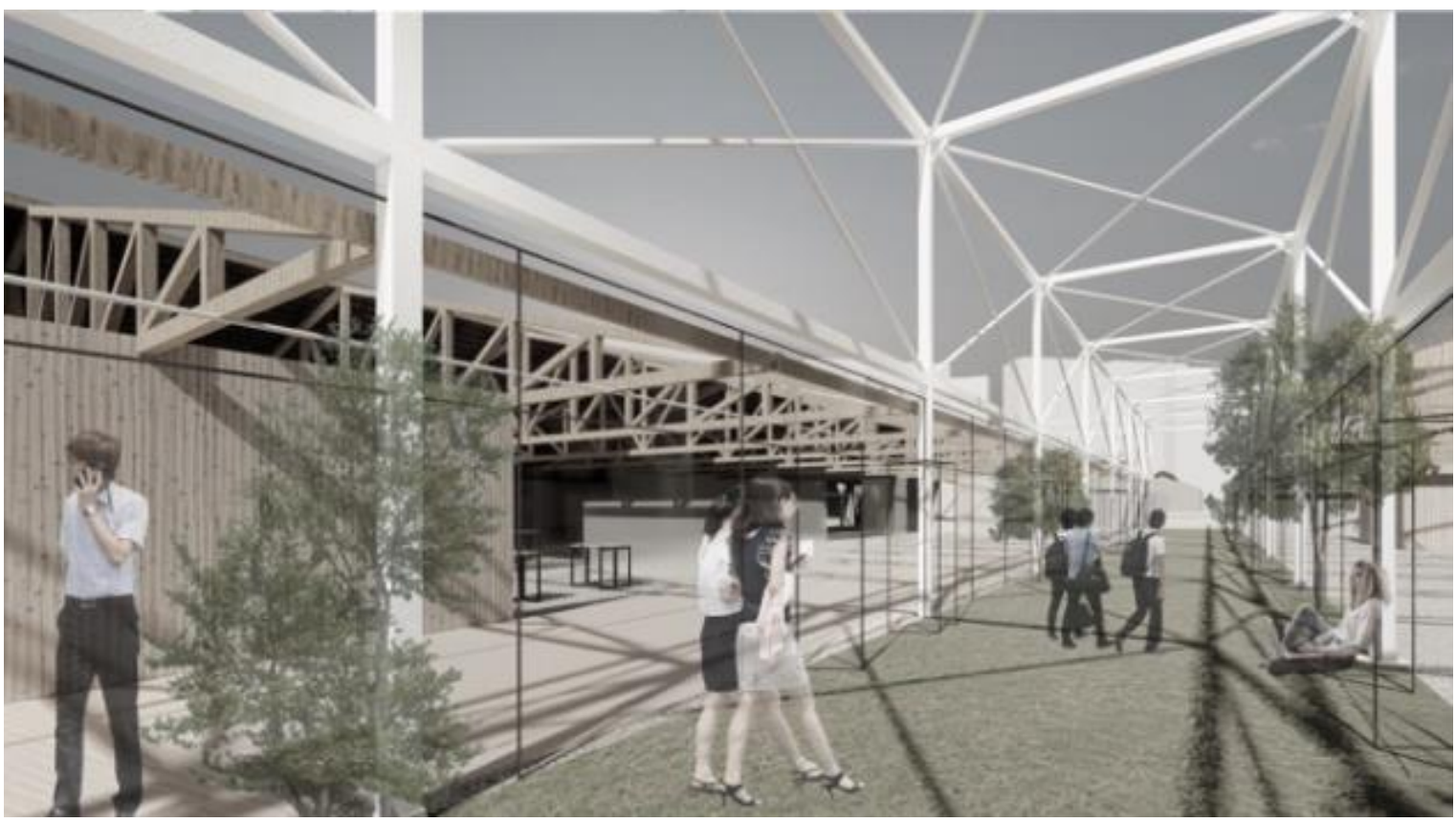

[Fig. 5] Expansion of Gaps by Overlapping

By emptying the roof in the factory interior space covered with a stuffy roof that did not receive sunlight, it expands the gap between the existing cramped gaps and introduces natural elements. As the interior space is transformed into the exterior space, external environmental elements permeate the interior space of the factory, allowing the overall space to be revived.

\subsubsection{Space between Adjacent Spaces}

This study proposes that the gap between the building and the adjacent space between the buildings is planned as a place to expand the spatial meaning of the gap. It is proposed as a place where people can feel the meaning of the gap spatially by planning it as a place of spatial experience where people can feel the meaning of the gap itself, centering on the empty spaces between the factories and the traces of the past. 


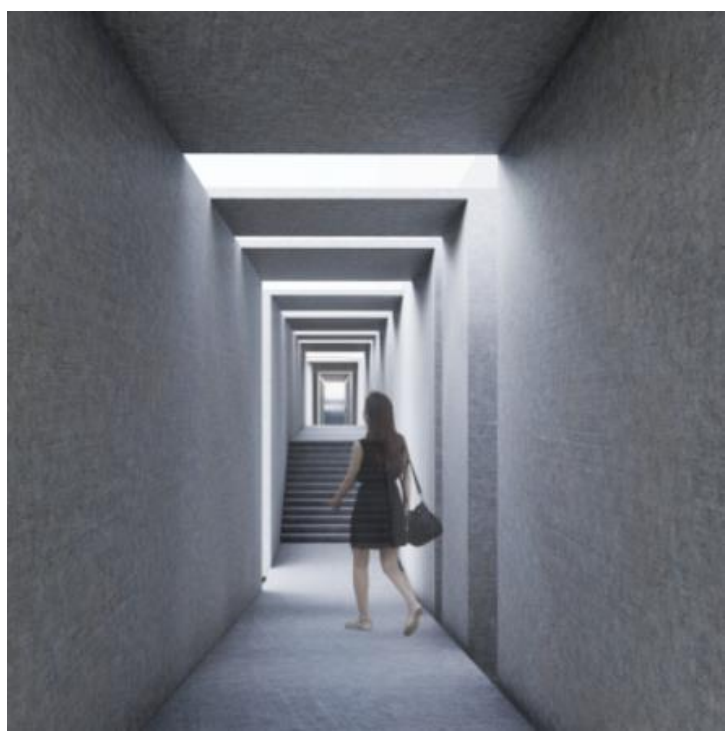

[Fig. 6] The Space of the Gap Experience Hall

\subsection{Overall View of the Proposal}

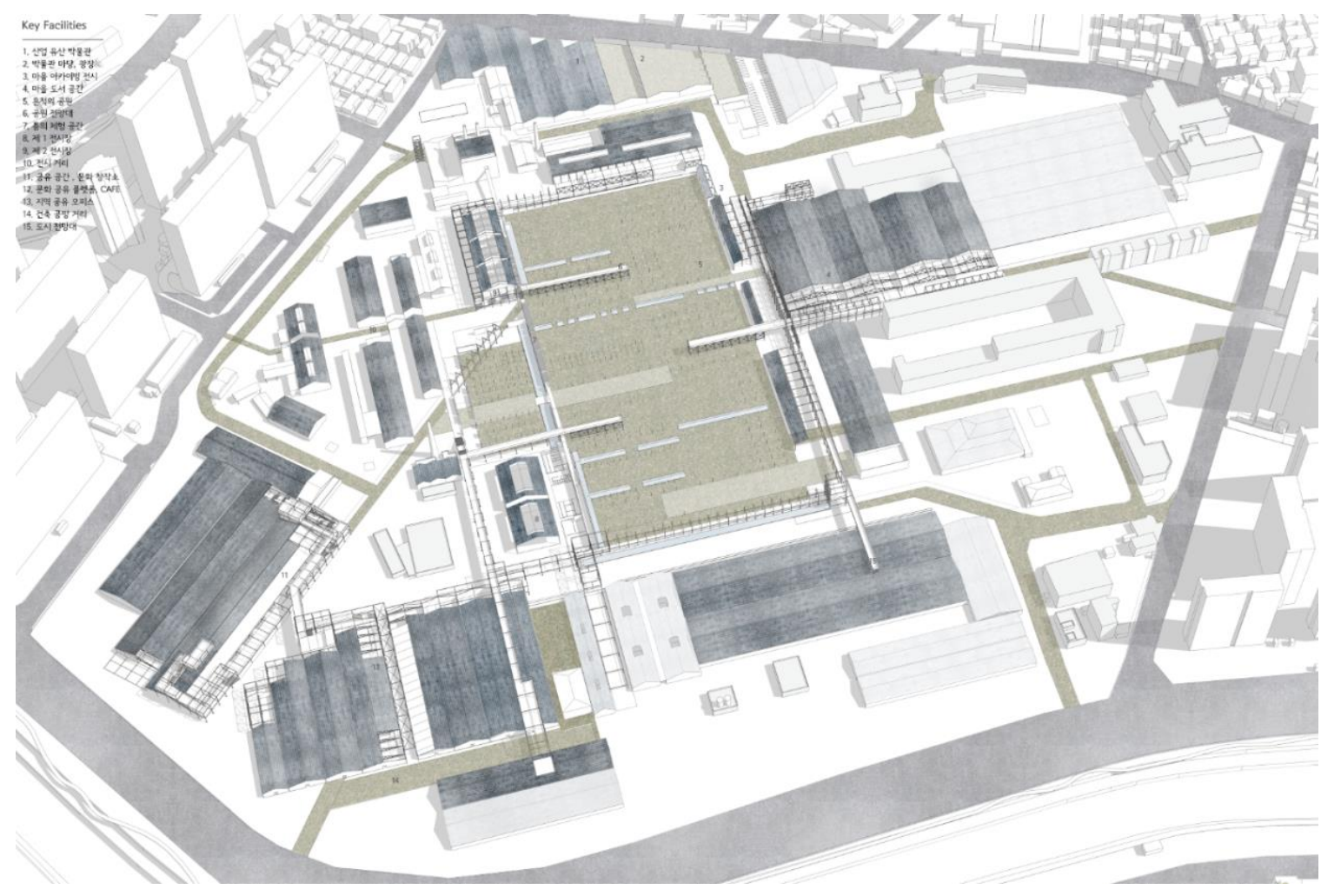

[Fig. 7] Overall View of the Proposal

The modern society is developing rapidly, and the spaces of the past are rapidly disappearing due to urban development. In this study, the industrial heritage spaces of Ilshin Textile Factory and Jeonnam Textile Factory is divided into the currently used site and the unused space, and divide the stages of the plan. The east site, where the logistics center, nursing hospital, and day care center are located, preserves the original space as much as possible, and the elevated water tank, warehouse, and 
restaurant located in the north can be used as the original space as a museum program. The site of the factory located to the west was used as an exhibition hall, and it is intended to be used as an architecture exhibition hall, space experience hall, etc. All the gaps located in the site are interwoven with each other, the space is expanded, and the roads of the gap are penetrated into the central park. As the empty gap space expands, the gap spaces of the factory are intertwined and combined with each other in the site, and are planned as architectural planning elements that activate the proposed site. Not only that, the elements of the gaps inside the site can be broadly extended to the urban space beyond the proposed site.
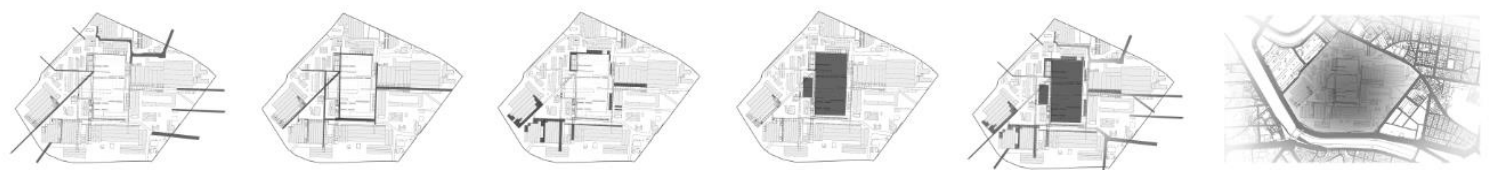

[Fig. 8] The Gap between Adjacent Spaces and Cities

By emptying the space at the center of the site, it was planned so that the spaces in all gaps could be gathered into the central space. As a result, the empty gap space and the expanded gap space gather at the center of the site and spread throughout the site. The roads in each gap are elements that connect the abandoned and utilized spaces of the site. At the same time, it acts as an element that connects the disconnected site with the road around the site. As a result, it can be urbanized within the proposed site.

\section{Conclusions}

The purpose of this study is to propose an architectural plan that can revitalize the space between gaps into a place with new value according to its typological characteristics. For this purpose, the concept and characteristics of boundaries and gaps between spaces were examined, and the typological characteristics of spaces were identified. Spatial types of gaps are divided into gaps created by overlapping spaces, gaps due to interlocking overlap, and gaps in adjacent spaces at a distant from each other. This study applied this spatial type of gaps as a gap between the space, a gap between the overlapping roofs, and the space between buildings in the proposed site.

This study proposes to spatially and functionally expand the potential of the space by paying attention to the space of the gap as a boundary space. The significance of this study lies in the idea that it does not simply extend the physical scale of the space, but suggests a planning method that can fill the temporal and spatial gaps of partially cut off urban spaces. Proposing a new cultural program by emptying and expanding the existing space of the gap caused by the overlapping of spaces and expanding the gap space caused by the overlapping roof by using a wire frame combined with the existing structure can be evaluated as methods of planning that change the physical properties of the gap space so that the gap space as a boundary space can play a central role in the new space. Planning the gap space between the factory buildings of the proposed site as a place of spatial experience where people can feel the meaning of the space itself through the traces of the past is to change the nonphysical properties of the gap space. This is a method of planning to expand the historical and cultural meaning of the gap space in a way that can revitalize the proposed site by weaving and combining the spaces within the factory site that were cut off from each other.

This study proposed an alternative plan that could revitalize the space with new value by paying attention to the space that was cut off in the process of rapid growth and development. Nevertheless, the fact that the proposal of the plan can be applied in a limited way to a specific site and that three spatial types of gaps are defined are the limitations of this study. Therefore, in order to derive a 
planning element based on the spatial characteristics of the gap, which can become the center of a new space, rather than an unnecessary negative space, follow-up research is needed by subdividing the typological characteristics of the gap space and proposing various alternatives that can turn it into an architectural space.

\section{References}

[1] Y. J. Jung, Study of Geometric Abstract through the Perspective Space, Ewha Womans University, Master's Thesis, pp.5-7, (1991)

[2] M. S. Lee, Study of Inner Psychological Expression through Space, Sungshin Women's University, Master's Thesis, pp.5-6, (2017)

[3] C. Y. Ji, G. G. Yoon, A Study on the Types of Niche' as the Border in the Space, KIID Autumn Conference Proceeding, Korea Institute of Interior Design, pp.181-185, (2009), October 30, Asan, Korea

[4] J. Y. Lee, A Study on the Interior and the Exterior in the Architectures of Wright, Mies, Le Corbusier, Aalto, Seen from the Point of View of the Significance of Limit and World, Journal of the Architectural Institute of Korea Planning \& Design, (2015), Vol.31, No.2, pp.215-223, DOI: http://dx.doi.org/10.5659/JAIK_PD.2015.31.2.215

[5] S. S. Choi, H. J. Kim, Y. H. Jeon, K. H. Kim, A Study on the Space of Threshold including the Other in Contemporary Architecture, Journal of the Architectural Institute of Korea, (2006), Vol.22, No.4, pp.179-186, UCI: G704A00167.2006.22.4.035

[6] S. M. Park, Y. S. Lee, A study on the Characteristics of the Blurred Zone in Contemporary Architecture's Boundaries, Proceeding of Autumn Annual Conference, Architectural Institute of Korea, pp.269-272, (2011), October 29, Gyeongsan, Korea.

[7] J. W. Kim, An Opening as a New Beginning of New Space, Ewha Womans University, Master's Thesis, pp.6-8, (2000)

[8] T. I. Kweon, A Study on the Feature of Transition Space of Modern Catholic Church Architecture with the Use of Space Syntax Analysis, Journal of the Korea Institute of Spatial Design, (2019), Vol.14 No.7, pp.73-86, DOI: http://dx.doi.org/10.35216/kisd.2019.14.7.73

[9] Y. R. Joo, J. E, Yoon, A Study on the Boundary Characteristics of Interior and Exterior in the Architectural Expression of Herzog \& de Meuron, Journal of Basic Design \& Art, (2019), Vol.20, No.2, pp.447-460, DOI: http://dx.doi.org/ 10.47294/KSBDA.20.2.33

[10] T. K. Han, A Study on the Fluctuation of Space as the Living System in Contemporary Japanese Architecture Focused on Works of Toyo Ito, SANAA, and Sou Fujimoto, Journal of the Korean Society Design Culture, (2016), Vol.22, No.3, pp.627-637, UCI: G704-001533.2016.22.3.016

[11] S. Y. Hwang, Spa Space Planning from the Perspective of Ambiguity of Borders and Middle Zone, Hongik University, Master's Thesis, pp.14-16, (2008)

[12] Im-dong, Buk-gu, Gwangju Metropolitan City, http://www.ilshin.co.kr/front/kor/company/sub_01_02.do, Jul 5, (2021) 